\title{
Magnetic Properties of Structural Steels for Simulation of Crack Monitoring by Finite Element Method
}

\author{
Menno Patrick van der Horst ${ }^{1,2}$ (D) Miroslaw Lech Kaminski ${ }^{1}$
}

Received: 22 January 2018 / Accepted: 24 November 2019 / Published online: 7 December 2019

(c) The Author(s) 2019

\begin{abstract}
The metal magnetic memory method is a novel technique for monitoring fatigue cracks in steel structures, which can reduce operational expenses and increase safety by minimizing inspections. The crack geometry can be identified by measuring the self magnetic flux leakage, which is induced by the Earth's magnetic field and the permanent magnetization. The finite element method can be used to simulate the induced magnetic field around cracks to help interpret the self magnetic flux leakage measurements, but it is unclear what material properties to use. This study aims to determine the magnetic permeability of structural steel for accurate simulation of the induced magnetic field around cracks by the finite element method. The induced magnetic field was extracted from measurements above two square steel plates, one without defect and one with a straight slit, and compared with finite element results in function of the relative permeability. For both plates, a uniform relative permeability could be found for which experimental and numerical results were in good agreement. For the plate without defect and a relative permeability of 350 , errors were within $20 \%$ and were concentrated around the plate's edges. For the plate with the slit and a relative permeability of 225 , errors were within $5 \%$.
\end{abstract}

Keywords Crack monitoring $\cdot$ Metal magnetic memory $\cdot$ Self magnetic flux leakage $\cdot$ Finite element method $\cdot$ Magnetic permeability $\cdot$ Permanent magnetization

\section{Introduction}

Ship and offshore structures are continuously loaded by waves during their service life. The cyclic nature of wave loading can cause fatigue cracks to initiate, grow, and propagate. Periodical inspection is necessary to detect fatigue cracks before they cause a structural failure. Once a crack has been detected that is not of a critical size yet, its propagation should be monitored. Typically, this is done by increasing the inspection frequency, which is costly and causes downtime. A real-time wireless crack monitoring

Menno Patrick van der Horst was affiliated with Delft University of Technology at the time of writing this publication.

Menno Patrick van der Horst

menno_vd_horst@hotmail.com

Miroslaw Lech Kaminski

m.1.kaminski@tudelft.nl

1 Delft University of Technology, Mekelweg 2, 2628 CD Delft, The Netherlands

2 Houston, TX, USA system could reduce operational expenses and downtime while increasing safety. The applicability of several nondestructive evaluation (NDE) methods on a crack monitoring system for marine structures was reviewed in [1].

The most promising method discussed in [1] is the metal magnetic memory (MMM) method, which is an advanced NDE method that can be used to detect or monitor flaws, such as cracks, in ferromagnetic materials [2-5]. This method is very similar to magnetic flux leakage (MFL) testing but does not require active magnetization of the specimen. Instead, the MMM method measures the self magnetic flux leakage (SMFL), which is induced only by the Earth's magnetic field and a permanent magnetization that is caused by the material's hysteresis property [6]. Developing an understanding of both the induced and permanent magnetic fields around a crack is necessary to interpret the SMFL measurements and determine the crack's geometry reliably. The permanent magnetization of a steel structure varies slowly in time and is affected by remanence [7], stresses [8-11], and temperature fluctuations [12, 13]. The induced magnetization can vary instantaneously and is influenced 
by the structure's geometry, the background field, and the steel's magnetic permeability.

The Earth induced magnetic field distribution around ferromagnetic structures with complex boundary geometries such as a steel plate with a fatigue crack can be simulated numerically by the finite element method (FEM). The accuracy of FEM has been demonstrated in [14] by comparing simulation results of a thin ferromagnetic plate in a uniform background field with its analytical approximation. Hwang and Lord were the first to use FEM to model the interaction between the magnetic field and a defect, which they demonstrated for a circular ferromagnetic bar with a rectangular surface defect [15]. Currently, there are several commercial FEM software packages available (e.g. ANSYS Maxwell and COMSOL Multiphysics) to simulate the interaction between magnetic fields and a wide variety of defect shapes, sizes, and locations likely to be seen in practice.

The aim of this paper is to determine the magnetic permeability of structural steels for accurate simulation of the induced magnetic field distribution around cracks using FEM. Steel's magnetic properties depend greatly on the microstructure (e.g. grain size, pearlite fraction, carbon content, manganese content) [16, 17], and are generally described by the hysteresis curve, which is the nonlinear relationship between the magnetic field strength and the magnetic flux density (B-H curve). However, for weak fields such as the Earth's magnetic field, the B-H curve can be assumed linear, see Fig. 1. The slope of the curve is the magnetic permeability resulting in the induced magnetization and a vertical offset can be caused by the permanent magnetization. Note that every grain or magnetic domain of the steel's microstructure can have a different B-H curve that may even be different for the $\mathrm{X}, \mathrm{Y}$, and $\mathrm{Z}$ directions.

To determine the magnetic permeability of a steel plate, induced magnetic field measurements are compared to finite element (FE) results for a number of values for the relative permeability. This way, the global permeability of an entire

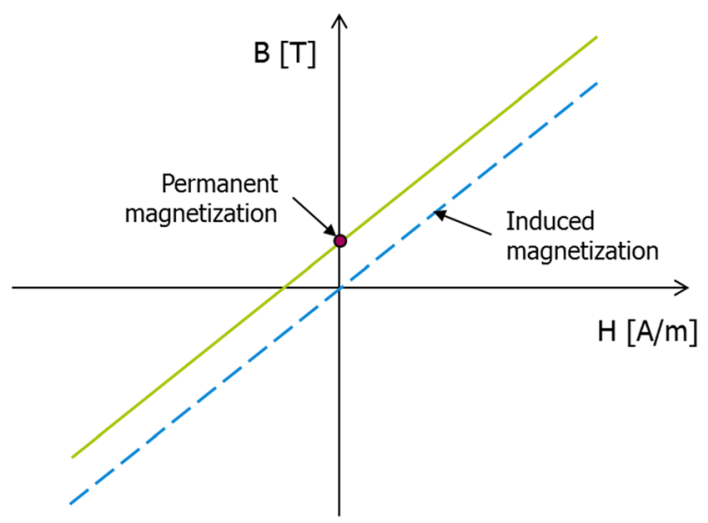

Fig. 1 Linear approximation of the hysteresis curve, after [14] plate can be determined, unlike in [18] where the permeability was determined of a small ellipsoid sample. The induced magnetic field is separated from the permanent magnetic field using two different methods. For a symmetric plate, the permanent magnetic field can be filtered out by repeating the measurements with the plate rotated 180 degrees. This is done in Experiment 1 for a square steel plate without defect. For an object of any other geometry, the permanent magnetic field can be filtered out by repeating the measurements in zero magnetic field by using a magnetic field simulator, which has been done in the past by researchers focusing on reducing the magnetic signature of naval ships [19, 20]. This is done in Experiment 2 for a square steel plate with a slit. The methods of both experiments, including their FE models, are explained in Sect. 2. The results are presented in Sect. 3 and discussed in Sect. 4. Finally, some conclusions are drawn.

\section{Method}

Two experiments were conducted. In the first experiment, magnetic measurements were taken above a steel plate without any defect while in the Earth's magnetic field. By extracting the induced magnetic field from the measurements and comparing it with a linear magneto-static FE model of the same plate, the average magnetic permeability of the plate could be determined. In the second experiment, the steel plate from Ref. [18] with a straight slit was held in a magnetic field simulator while taking magnetic measurements above the slit. Again, the induced magnetic field was extracted from the measurements to be compared with FE results in order to find the magnetic permeability of the plate. Both experiments and their FE models are described in more detail in the subsections below.

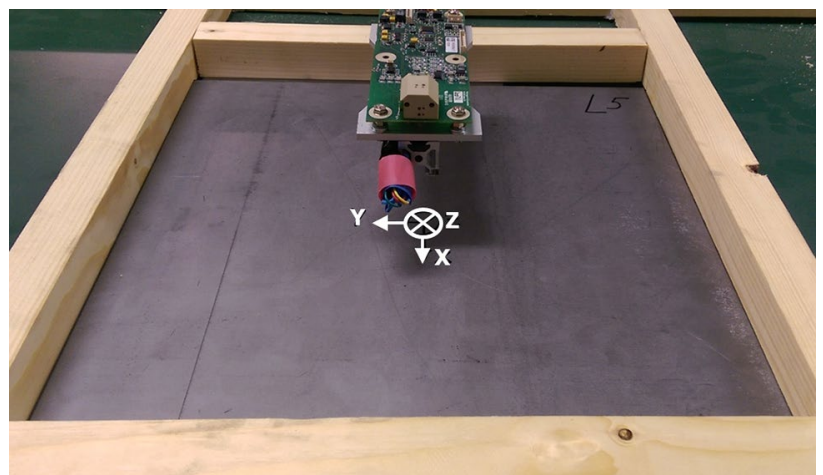

Fig. 2 Experimental setup Experiment 1 


\subsection{Experiment 1}

A square FeE235 steel plate of $300 \mathrm{~mm}$ wide and $5 \mathrm{~mm}$ thick is placed underneath a 3-axis fluxgate magnetometer with nanoTesla sensitivity that can be moved in space in a

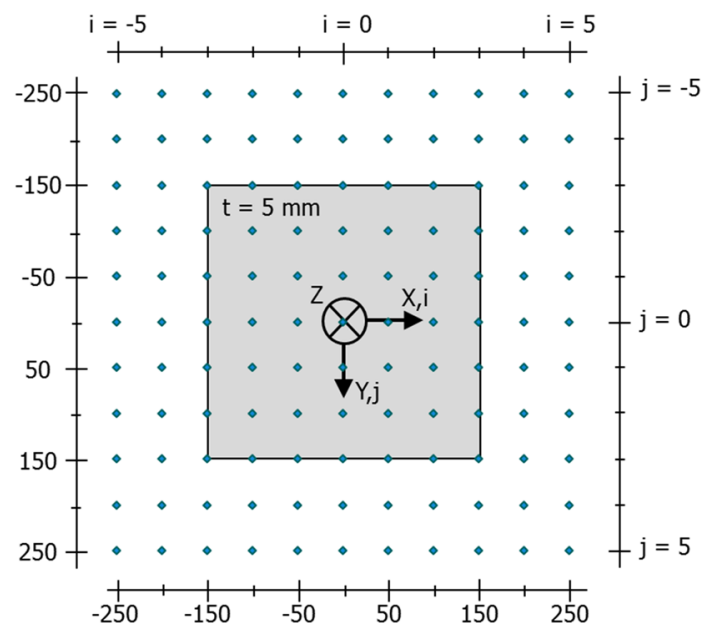

Fig. 3 Test plate Experiment 1 with measurement grid
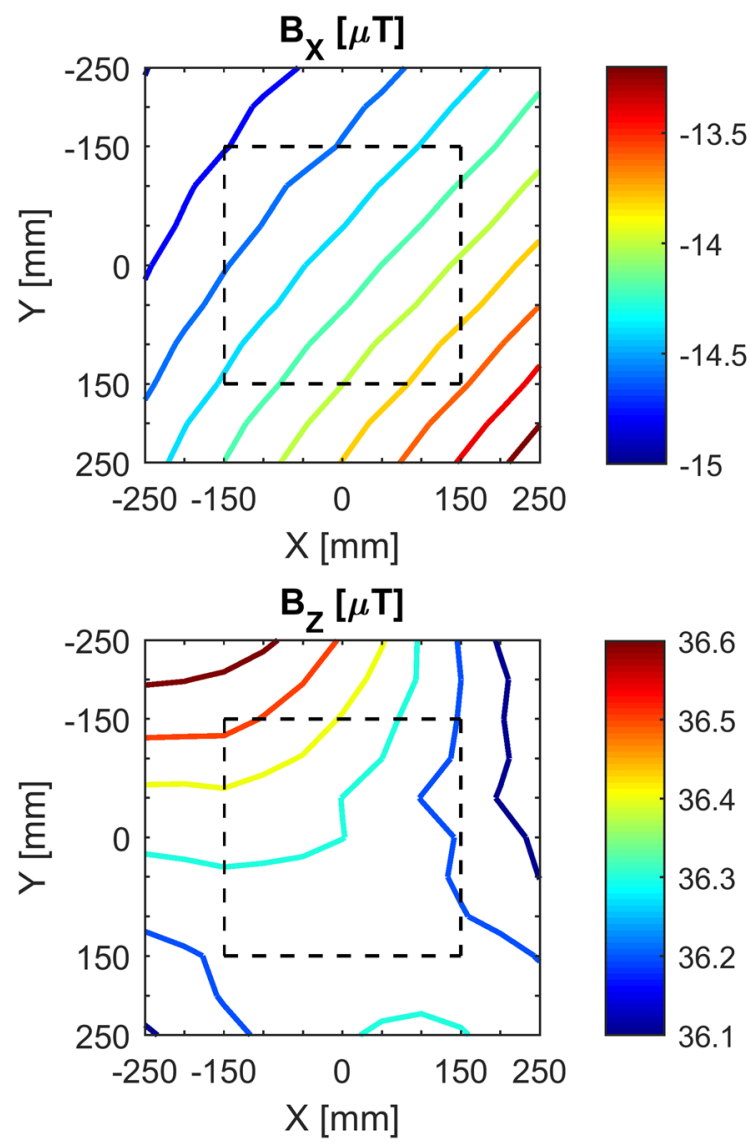

controlled way, see Fig. 2. The sensor is programmed to take measurements on a grid of 121 points $75 \mathrm{~mm}$ above the plate surface, see Fig. 3, which is the closest distance this test setup allows. Note that this setup was designed for plates in horizontal orientation. At every grid point, the sensor takes 10 measurements in exactly one second, which are then averaged. The grid columns are numbered from $i=-5$ to $i=5$ and the rows are numbered from $j=-5$ to $j=5$. Note that this measurement grid $(i, j)$ is not associated to the steel plate, but to the measurement environment. The measurements are repeated with the plate rotated 180 degrees around the Z-axis to be able to filter out the permanent magnetic field. The same setup is also used to measure the background field with the plate removed.

\subsection{FE Model 1}

Experiment 1 is simulated by a linear magneto-static FE model using ANSYS Maxwell. The steel plate is assumed to have no permanent magnetization, so its magnetization is dependant only on the relative permeability $\mu_{r}$, the background field $B_{B G}$, and the plate's geometry. The geometry of the plate is known and the background field has been
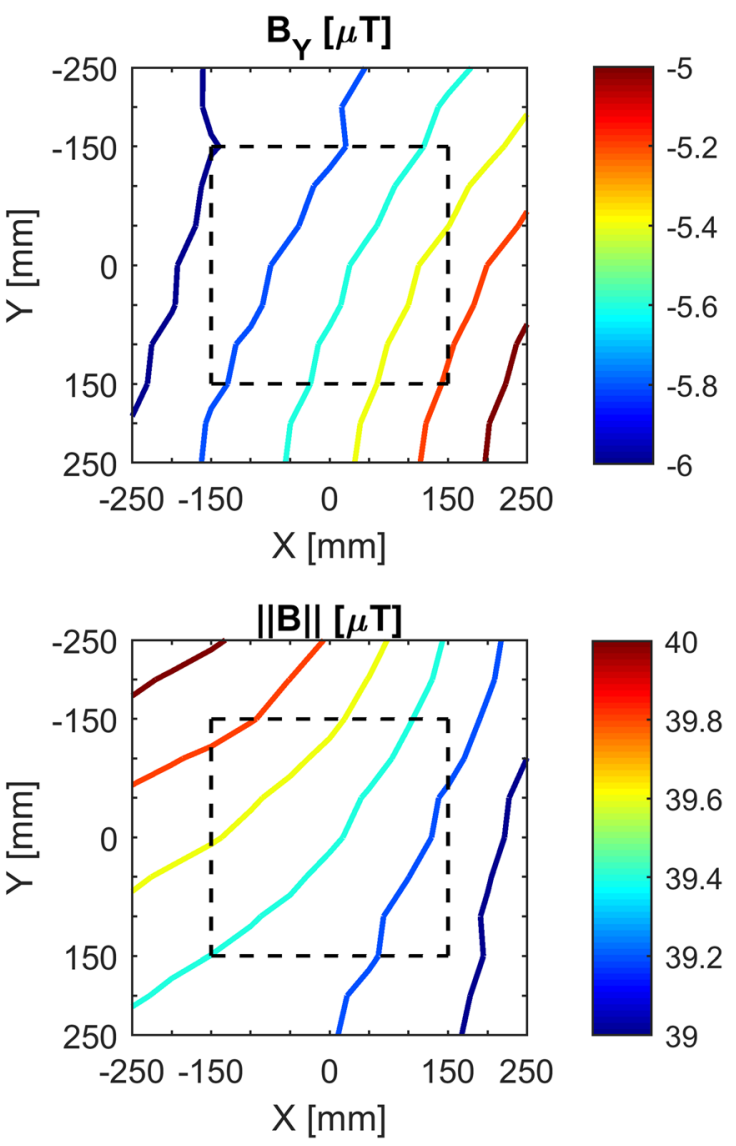

Fig. 4 Measured background field 
measured using the test setup of Experiment 1 without a steel plate. The measured background field can be seen in Fig. 4. Note that the dashed square shows the location where the plate will be placed for the actual measurements. The measured background field is fairly uniform, so a uniform background field is assumed in the FE model as the average measured background field:

$B_{B G}=\left[B_{B G, X} ; B_{B G, Y} ; B_{B G, Z}\right]=[-14.3 ;-5.6 ; 36.3] \mu T$.

The model is meshed using linear tetrahedral elements with a minimum of two elements over the thickness of the plate. The background field is applied as boundary conditions on the outer surfaces of the model domain, which is a cube ten times the size of the plate. The domain is modeled as a vacuum, so $\mu_{r}=1$, which is a good approximation of the permeability of air. The relative permeability of the steel plate is set as a parameter that varies between 50 and 500 with steps of 50. A 3D representation of the FE model with the applied background field as a vector plot can be seen in Fig. 5. It can be observed that the background field is mostly in out-of-plane direction with respect to the steel plate, which makes this experimental setup not ideal for maximizing the magnetic flux density in the plate.

\subsection{Experiment 2}

A square FeE235 steel plate of $300 \mathrm{~mm}$ wide, $5 \mathrm{~mm}$ thick, and with a wire cut slit of $150 \mathrm{~mm}$ long and $0.3 \mathrm{~mm}$ wide, see Fig. 6, is put in a magnetic field simulator with

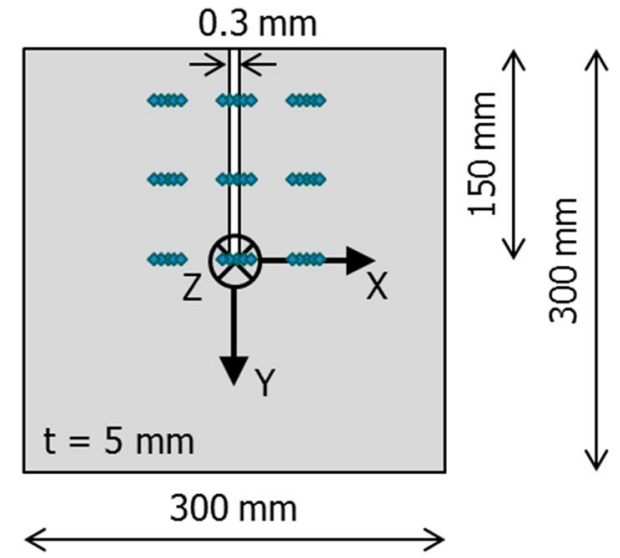

Fig. 6 Test plate Experiment 2 with measurement grid

Helmholtz coils in all three directions. One 3-axis fluxgate magnetometer with nanoTesla sensitivity and a sampling rate of $1000 \mathrm{~Hz}$ is used to measure the magnetic field above the plate while a second magnetometer measures the background field, as depicted in Fig. 7. An acrylic glass (polymethyl methacrylate) plate with a grid of holes is placed over the steel plate in order to fix the location of the sensor above the plate with pins.

Magnetic measurements are taken in 9 clusters of 5 points near the slit, each $30 \mathrm{~mm}$ above the steel plate, see Fig. 6. Note that the measurement grid shown in Fig. 6 depicts the approximate sensor locations and that the pickup coils for $\mathrm{X}, \mathrm{Y}$, and $\mathrm{Z}$ are spaced $15 \mathrm{~mm}$ from each other within the fluxgate magnetometer.
Fig. 5 FE model 1 with background field

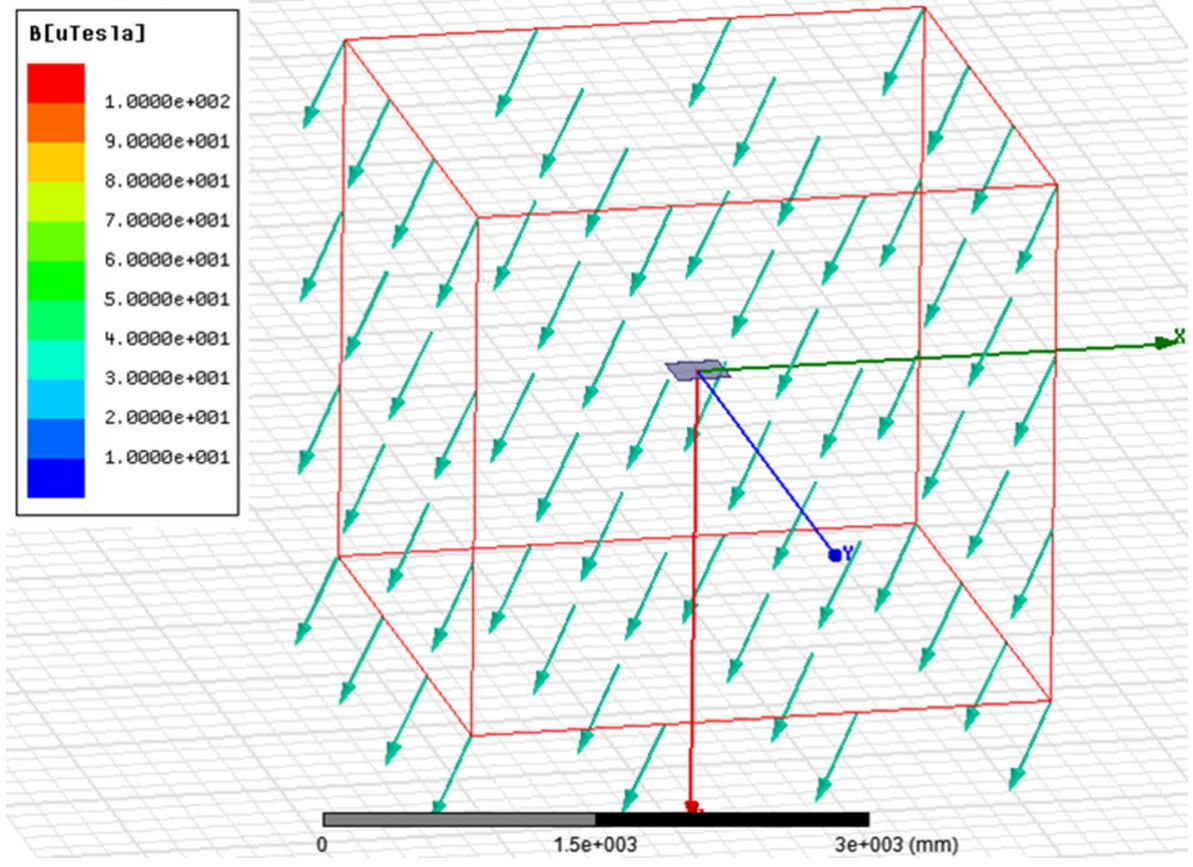




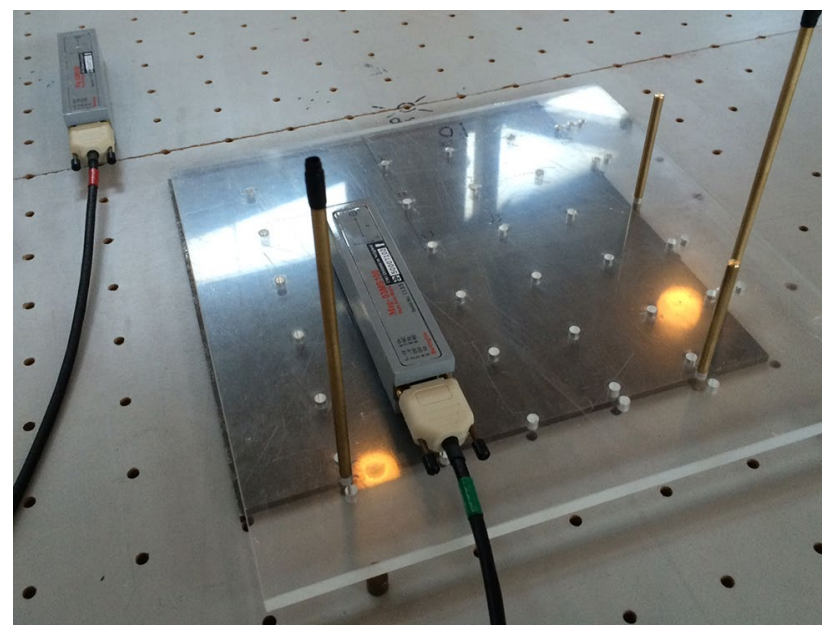

Fig. 7 Test setup Experiment 2

The first set of measurements is taken with a background field of $50 \mu \mathrm{T}$ (average Earth's magnetic field strength) in positive X-direction to maximize the flux leakage around the slit. The second set of measurements is taken with zero background field by counteracting the Earth's magnetic field with Helmholtz coils in 3 directions to determine the permanent magnetic field around the plate.

\subsection{FE Model 2}

Experiment 2 is also simulated by a linear magneto-static FE model using ANSYS Maxwell. Again, the steel plate is assumed to have no permanent magnetization, so its magnetization is dependant only on the relative permeability $\mu_{r}$, the background field $B_{B G}$, and the plate's geometry. The geometry of the plate is known, see Fig. 6. The background field is controlled in the experiment using a magnetic field simulator and is set on $50 \mu \mathrm{T}$ in positive X-direction. The same uniform background field is applied in the FE model:

$B_{B G}=\left[B_{B G, X} ; B_{B G, Y} ; B_{B G, Z}\right]=[50 ; 0 ; 0] \mu T$.

The model is meshed using linear tetrahedral elements with a minimum of two elements over the thickness of the plate and with a local mesh refinement around the slit, see Fig. 8, and around the measurement grid, so $30 \mathrm{~mm}$ above the plate surface.

The background field is applied as boundary conditions on the outer surfaces of the model domain, which is a vacuum cube ten times the size of the plate. The relative permeability of the steel plate is set as a parameter that varies between 50 and 500 with steps of 50. A 3D representation of the FE model with the background field as a vector plot can be seen in Fig. 9.

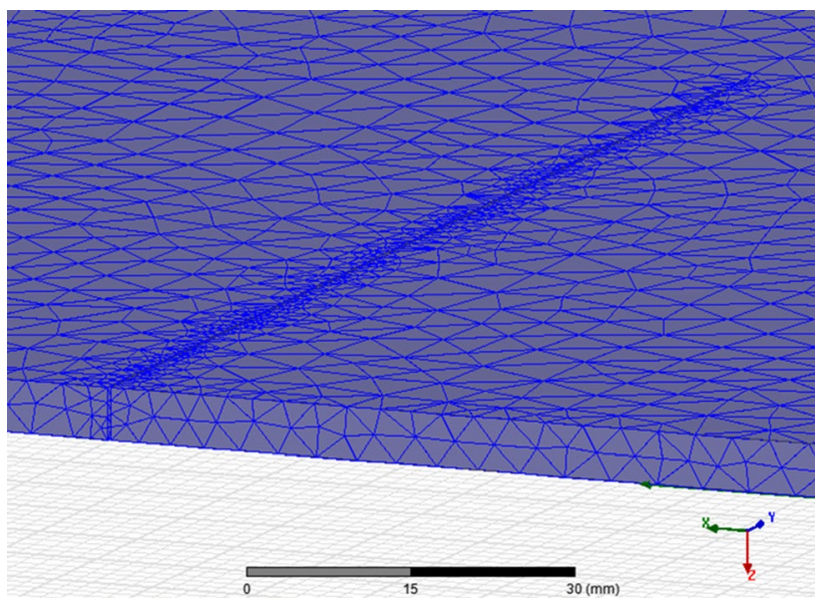

Fig. 8 FE model 2 local mesh refinement around slit

\section{Results}

\subsection{Experiment 1}

The first set of measurements with the plate as shown in Fig. 3 results in a total magnetic field as depicted in Fig. 10. The second set of measurements with the plate rotated 180 degrees around the $\mathrm{Z}$-axis results in a total magnetic field as depicted in Fig. 11. In both figures, the grey square shows the location of the steel plate.

When no stresses in the material, the total magnetic field consists of the background field, induced field, and permanent field. First, the background field is subtracted from the total field measurements to obtain the reduced fields:

$\vec{B}_{r e d, 1}(i, j)=\vec{B}_{t o t, 1}(i, j)-\vec{B}_{B G}(i, j)$,

$\vec{B}_{r e d, 2}(i, j)=\vec{B}_{t o t, 2}(i, j)-\vec{B}_{B G}(i, j)$,

for $i=-5, \ldots, 5$ and $j=-5, \ldots, 5$.

Now, the reduced fields $\vec{B}_{r e d, 1}$ and $\vec{B}_{r e d, 2}$ consist of an induced part and a permanent part:

$\vec{B}_{r e d, 1}(i, j)=\vec{B}_{r e d, 1}^{i n d}(i, j)+\vec{B}_{r e d, 1}^{p e r}(i, j)$,

$\vec{B}_{r e d, 2}(i, j)=\vec{B}_{r e d, 2}^{i n d}(i, j)+\vec{B}_{r e d, 2}^{p e r}(i, j)$,

for $i=-5, \ldots, 5$ and $j=-5, \ldots, 5$.

When rotating the plate 180 degrees around the Z-axis, the permanent magnetization rotates along and the induced magnetization stays the same. Note that this is only valid for $B_{X}$ and $B_{Y}$. Also, the induced magnetization is symmetric in $i$ and symmetric in $j$. This yields: 
Fig. 9 FE model 2 with background field
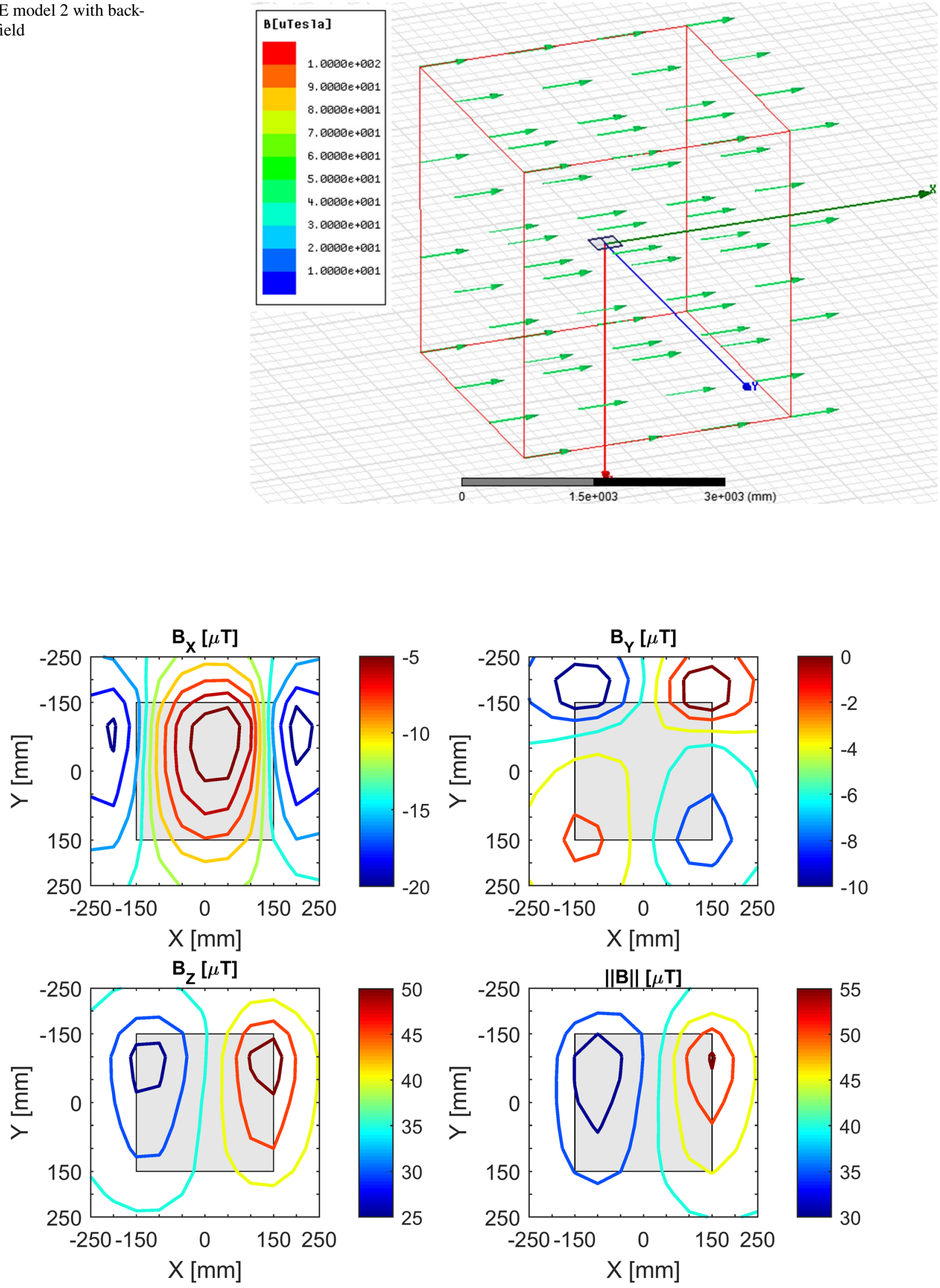

Fig. 10 Experiment 1 -total magnetic field $B_{t o t, 1} 75 \mathrm{~mm}$ above the plate surface 

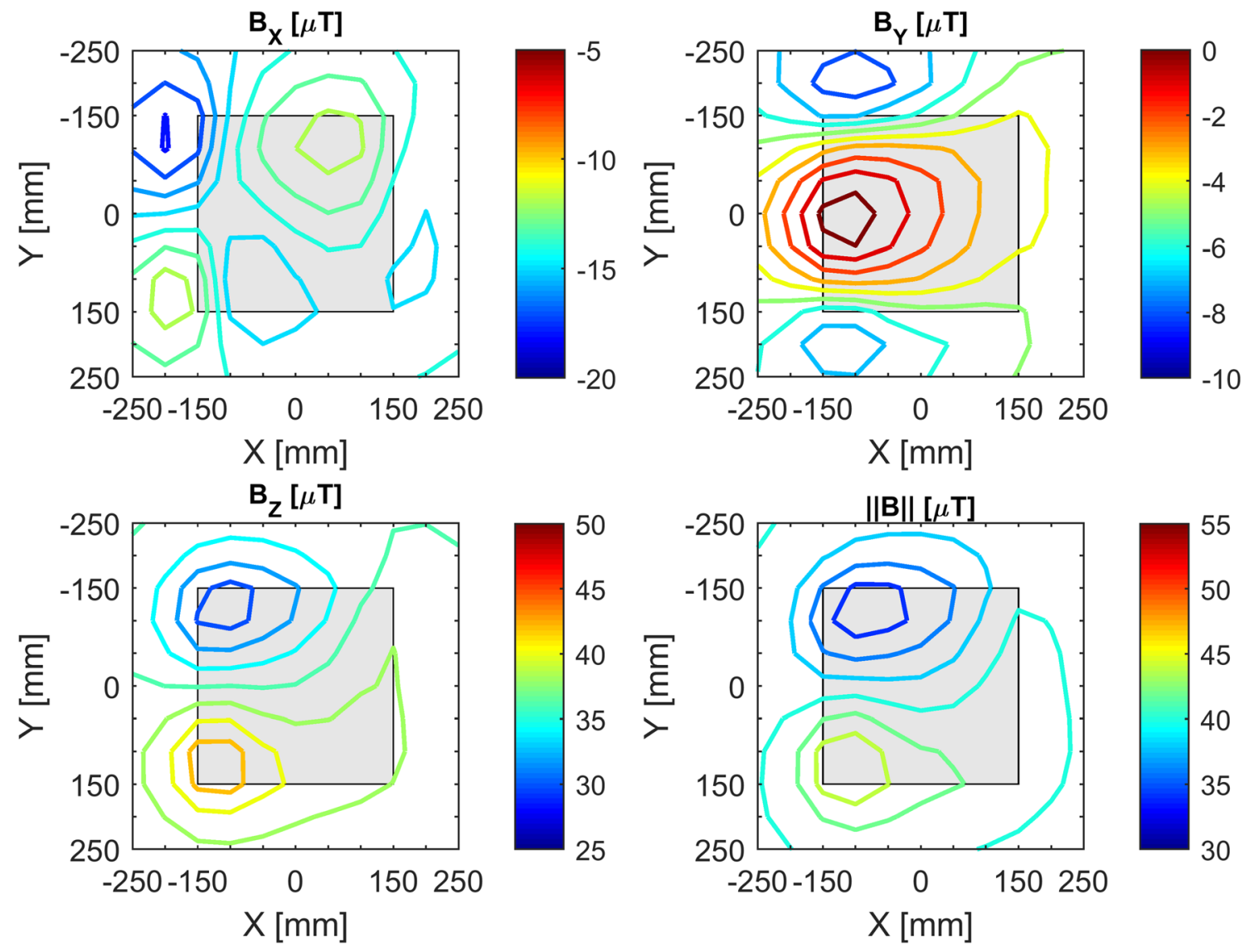

Fig. 11 Experiment 1 -total magnetic field $B_{t o t, 2} 75 \mathrm{~mm}$ above the plate surface
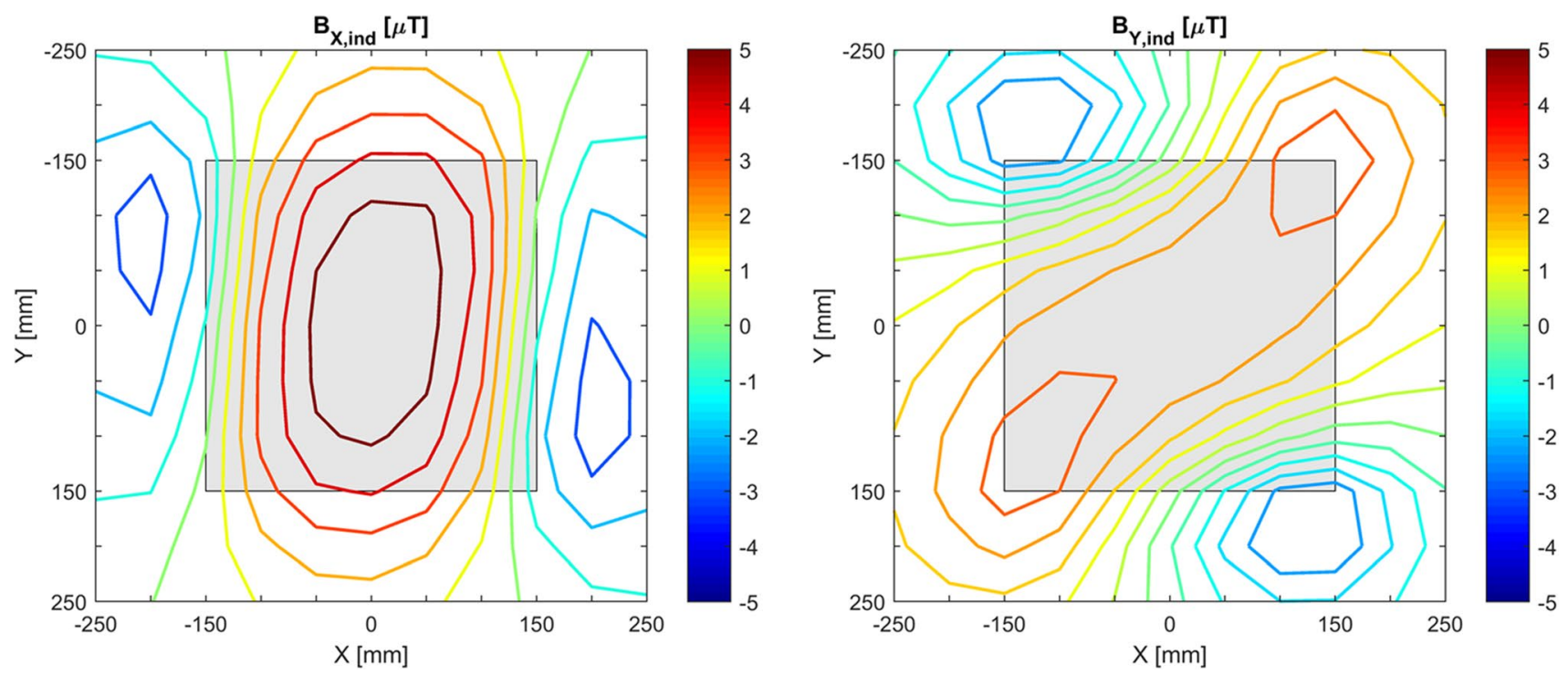

Fig. 12 Experiment $1-$ measured induced magnetic field $75 \mathrm{~mm}$ above the plate surface

$$
\begin{aligned}
& \vec{B}^{\text {ind }}(i, j)=\vec{B}_{\text {red }, 1}^{\text {ind }}(i, j)=\vec{B}_{r e d, 2}^{\text {ind }}(i, j)=\vec{B}_{r e d, 2}^{\text {ind }}(-i,-j), \quad(5) \quad \vec{B}^{p e r}(i, j)=\vec{B}_{r e d, 1}^{\text {per }}(i, j)=-\vec{B}_{r e d, 2}^{\text {per }}(-i,-j), \\
& \text { for } i=-5, \ldots, 5 \text { and } j=-5, \ldots, 5 .
\end{aligned}
$$



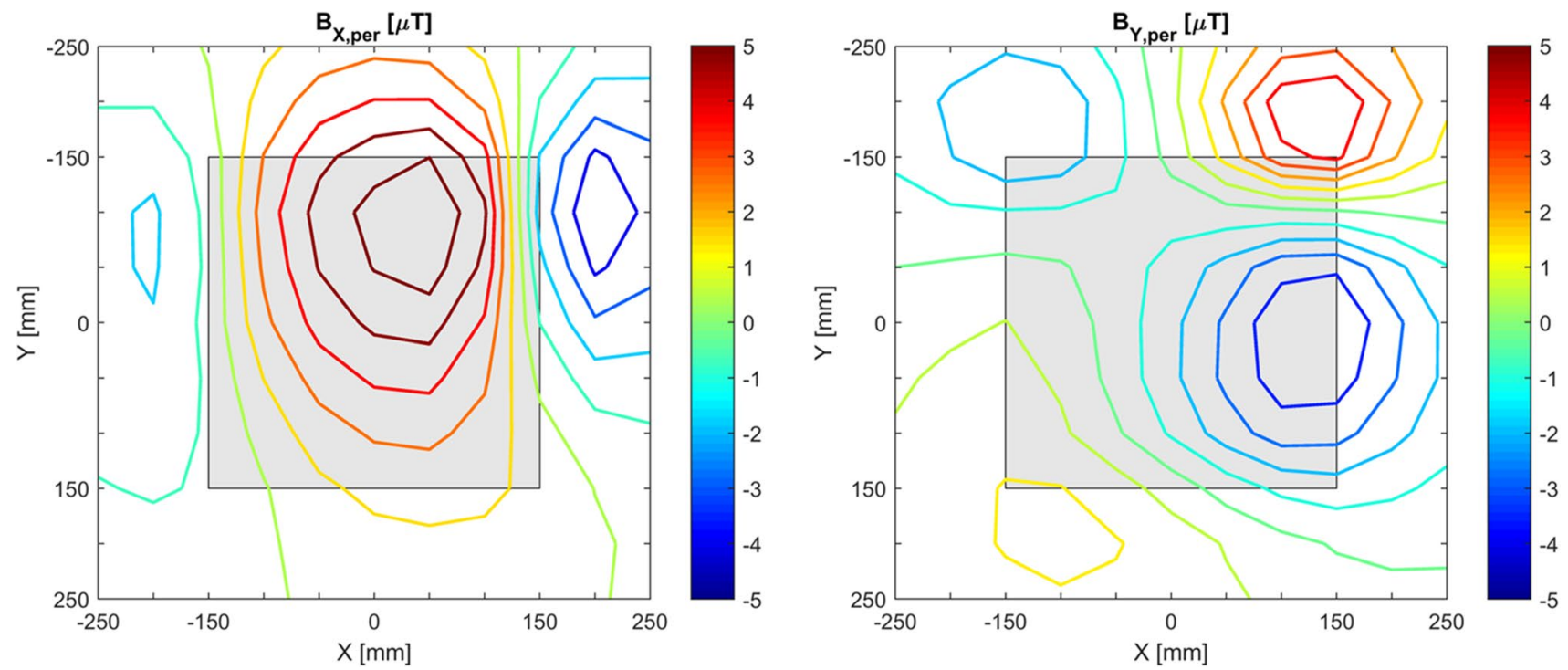

Fig. 13 Experiment 1 -measured permanent magnetic field $75 \mathrm{~mm}$ above the plate surface

Finally, combining Eqs. 3-6 results in the following equations for the induced and permanent magnetic fields:

$\vec{B}^{i n d}(i, j)=\frac{\vec{B}_{r e d, 1}(i, j)+\vec{B}_{r e d, 2}(-i,-j)}{2}$,

$\vec{B}^{p e r}(i, j)=\frac{\vec{B}_{r e d, 1}(i, j)-\vec{B}_{r e d, 2}(-i,-j)}{2}$,

for $i=-5, \ldots, 5$ and $j=-5, \ldots, 5$.

The resulting induced and permanent magnetic fields are shown in Figs. 12 and 13 respectively. Note that by rotation around the Z-axis only the permanent parts of the inplane components of the field distribution $B_{X}$ and $B_{Y}$ can be eliminated.

\subsection{Comparison with FE Model 1}

Now that the induced magnetic field has been extracted from the measurements of Experiment 1, it can be compared with the FE results in function of the relative permeability of the steel plate. This involves obtaining $B_{X}$ and $B_{Y}$ from the FE model in all 121 points of the measurement grid for each relative permeability and plotting the deviations from the experimental values by calculating the root mean squared error (RMSE) as follows:

$R M S E=\sqrt{\frac{1}{121} \sum_{k=1}^{121}\left[B_{\text {ind }}^{F E M}(k)-B_{\text {ind }}^{E x p}(k)\right]^{2}}$.
The results are shown in Fig. 14. It can be seen from the RMSE distributions that the best agreement occurs at $\mu_{r}=350$ for $B_{X}$ and at $\mu_{r}=450$ for $B_{Y}$, which are marked in red as the minimum RMSE values. Since the background field is approximately three times larger in $\mathrm{X}$-direction than in Y-direction, the curve for $B_{X}$ will likely be more reliable than for $B_{Y}$, so the relative permeability is likely to be closer to 350 . The valleys in Fig. 14 are not very pronounced, especially for $B_{Y}$, which is likely due to the limitations of the experimental setup regarding the large lift-off distance between sensor and plate surface, and the small in-plane components of the background field.

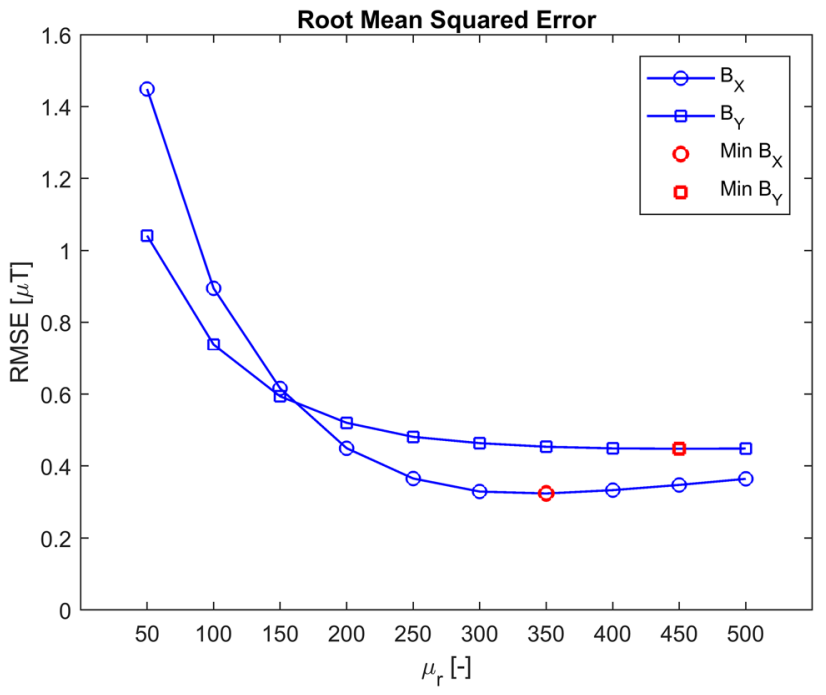

Fig. 14 Experiment 1-RSME between measurements and FE results 

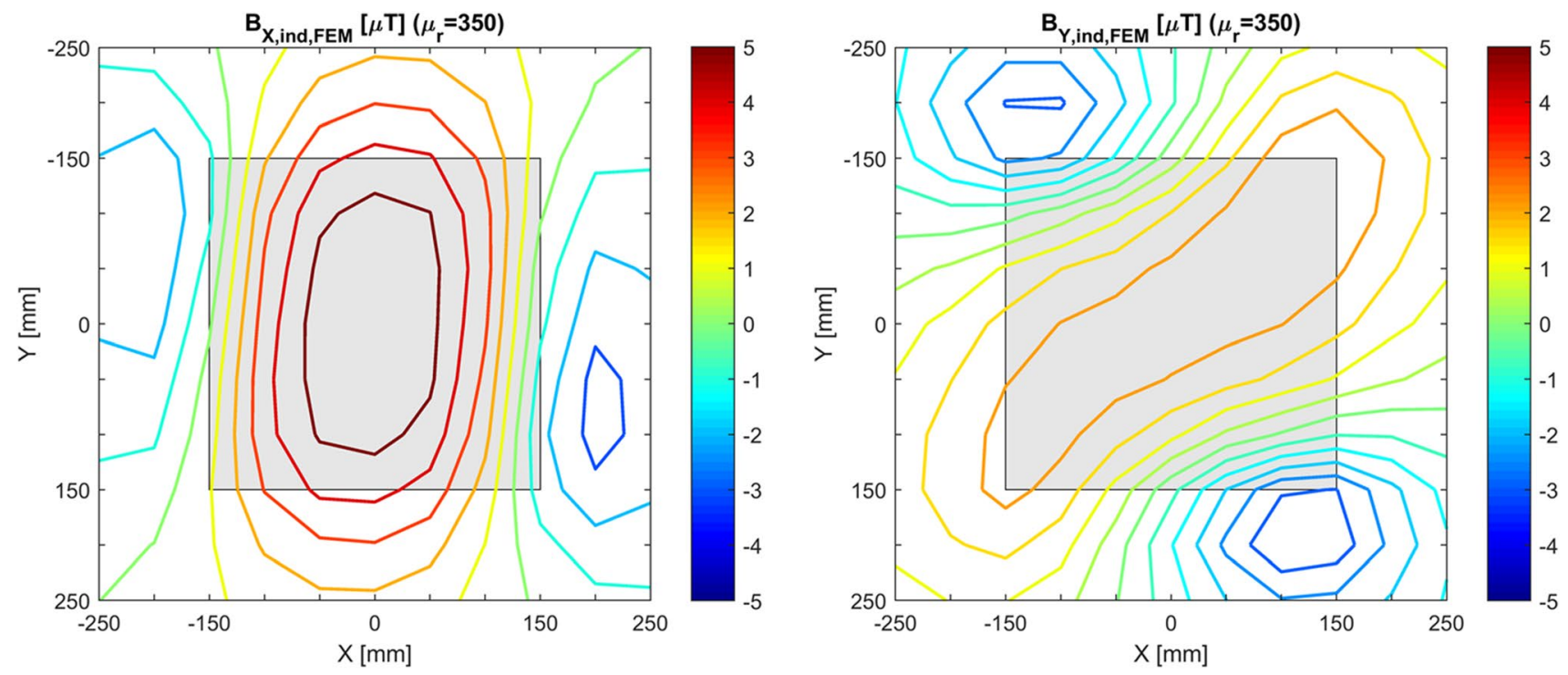

Fig. 15 Experiment 1-FE results induced magnetic field $75 \mathrm{~mm}$ above the plate surface

To illustrate the similarity between the measurements and FE results, the FE results for $\mu_{r}=350$ are shown in Fig. 15, which are in good agreement with the experimental results from Fig. 12 despite any measurement errors.

\subsection{Experiment 2}

The magnetic field measurements above the plate with a slit while in a background field of $50 \mu \mathrm{T}$ in X-direction applied by the magnetic field simulator are shown in Fig. 16. The figure shows both the position of the plate in grey and the measurement domain. Note that only the values in $\mathrm{X}$ and $\mathrm{Z}$-direction are shown because the

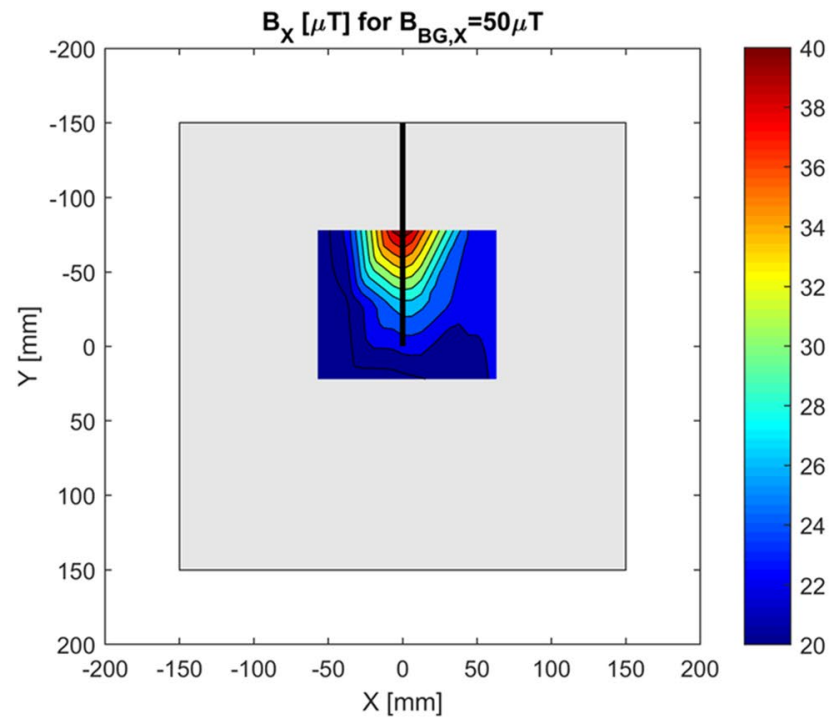

background field is in X-direction and the magnetic flux leakage signal is in Z-direction.

To be able to separate the induced and permanent magnetic fields, the measurements are repeated while the magnetic field simulator creates a zero field condition around the plate. Consequently, this set of measurements results in the permanent magnetic field distribution, see Fig. 17. Subtracting the permanent field from the total field results in the induced magnetic field, see Fig. 18.

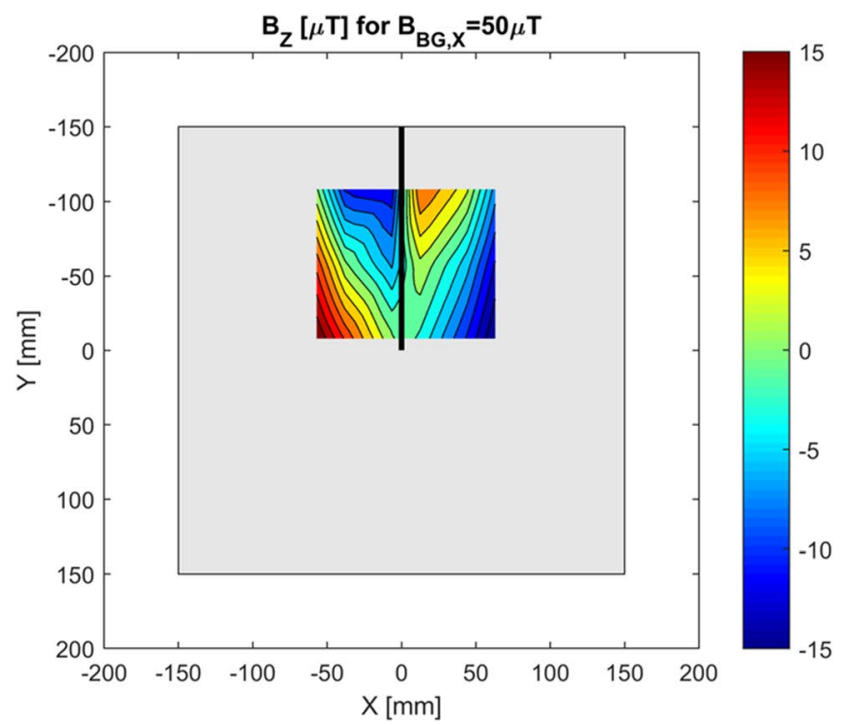

Fig. 16 Experiment 2-measured total magnetic field $30 \mathrm{~mm}$ above the plate surface 

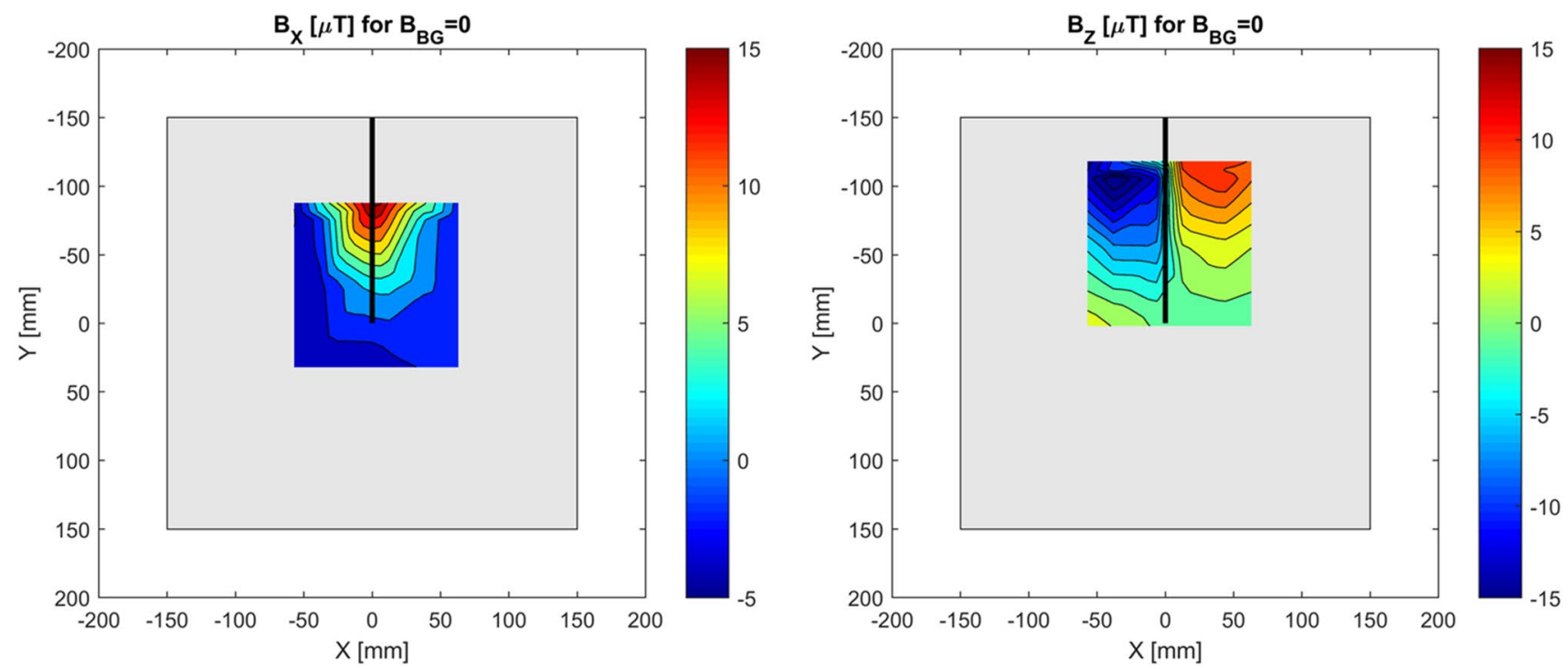

Fig. 17 Experiment 2-measured permanent magnetic field $30 \mathrm{~mm}$ above the plate surface

\subsection{Comparison with FE Model 2}

The FE model described in Sect. 2.4 is analyzed with a relative permeability of the steel plate ranging from 50 to 500 with a step size of 50. For each solution, $B_{X}$ and $B_{Z}$ are obtained in the 45 points of the measurement grid $30 \mathrm{~mm}$ above the plate surface. To show the deviation between measurements and FE results, the Root Mean Squared Error (RMSE) is calculated according to Eq. 9 with $n=45$.

The resulting RMSE distributions are shown in Fig. 19. It can be seen from the RMSE distributions for both $B_{X}$ and $B_{Z}$ that the best agreement is for a relative permeability between 200 and 250. By running an additional simulation, it is shown that the minima for both RMSE curves, which are marked in red, occur for a relative permeability of 225 .

To illustrate the similarity between the measurements and FE results, the FE results for $\mu_{r}=225$ are shown in Fig. 20, which are almost identical to the experimental results in Fig. 18.

\section{Discussion}

Considering the great similarity between measurements and FE results for both experiments, the presented separation techniques of induced and permanent magnetic fields are
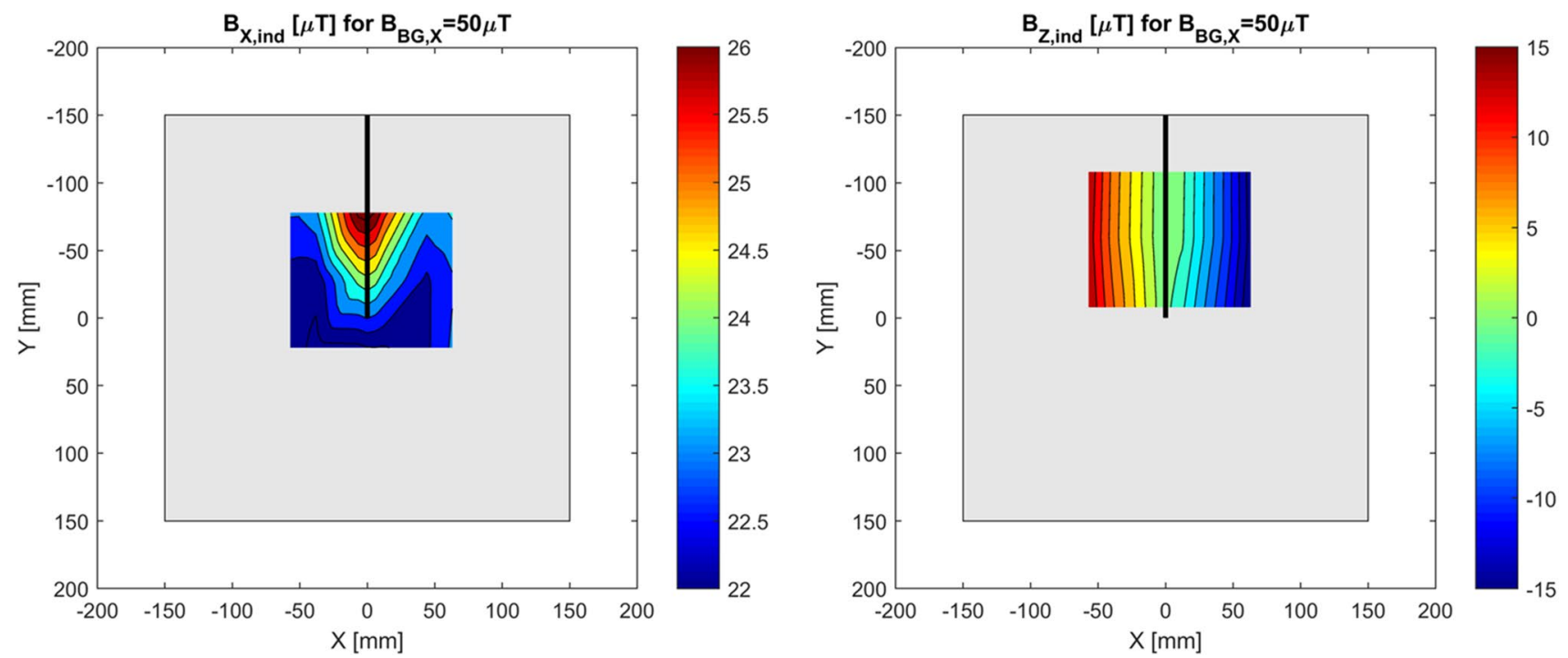

Fig. 18 Experiment 2-measured induced magnetic field $30 \mathrm{~mm}$ above the plate surface 


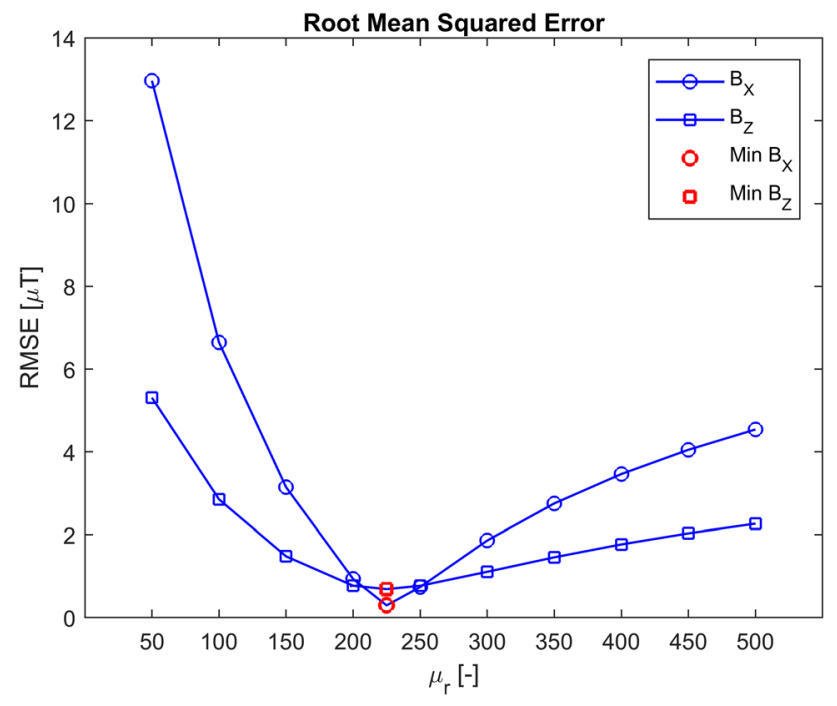

Fig. 19 Experiment 2-RMSE between measurements and FE results

successful. From the comparison of the measured induced magnetic field with FE results for varying $\mu_{r}$, it follows that the plate from Experiment 1 has a relative permeability of approximately 350 and that the plate with a slit from Experiment 2 has a relative permeability of approximately 225 . An important assumption is that the relative permeability of the steel plates is uniformly distributed and isotropic. To get more insight in the distribution of the material properties, the error distributions between measurements and FE results for Experiment 1 and Experiment 2 are shown in Figs. 21 and 22 respectively as a percentage of the maximum absolute measured value.

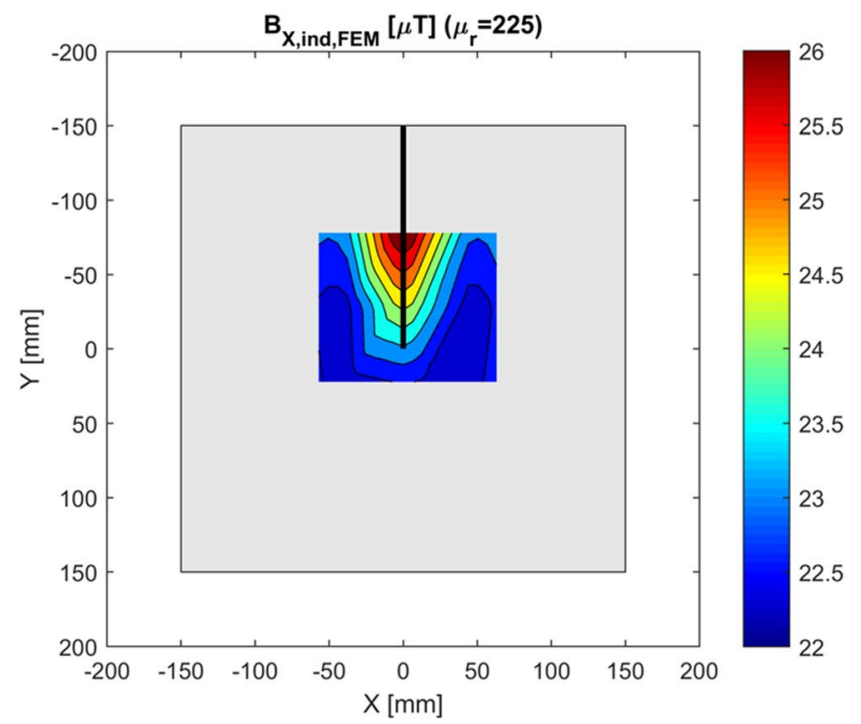

Experiment 1 shows much larger errors than Experiment 2 , which is most likely due to the large distance between the measurement domain and the plate and the relatively poor precision of the automatic sensor positioning system, both resulting in larger measurement errors. As could be expected, the largest errors for Experiment 1 are along the plate's edges where the magnetic field has the largest spatial gradient. The magnetic field in X-direction is expected to deviate most near the left and right edges and the field in Y-direction is expected to deviate most near the top and bottom edges, which is confirmed by Fig. 21. The smaller errors away from the plate's edges indicate that the relative permeability is likely to be uniformly distributed as was assumed in the FE model.

Figure 22 shows that for Experiment 2 the deviations between FE results and measurements are well within 5\% of the maximum absolute measured value for a relative permeability of 225 for the steel plate. The small errors indicate that the separation technique using the magnetic field simulator is successful and that a uniform and isotropic relative permeability of 225 may be assumed for this steel plate. Previous research in which a sample was wire cut from the exact same steel plate and analyzed concluded that the relative permeability is approximately 115 [18]. The difference between these results could mean that the sample manufacturing process had a significant effect on the magnetic material properties of the sample.

The measured permanent magnetic field distribution in Experiment 1 shows that the permanent magnetization of a steel plate can be non-uniformly distributed in an unpredictable manner, see Fig. 13. The permanent magnetic field shows an unexpected local concentration above the top right corner of the plate. Therefore, it is challenging to determine

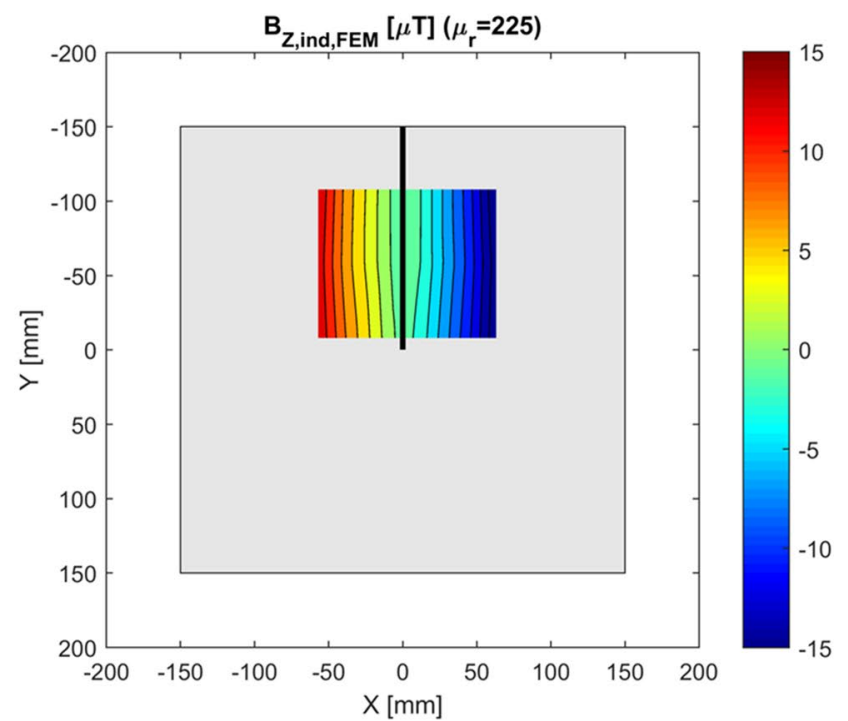

Fig. 20 Experiment 2-FE results induced magnetic field $30 \mathrm{~mm}$ above the plate surface 

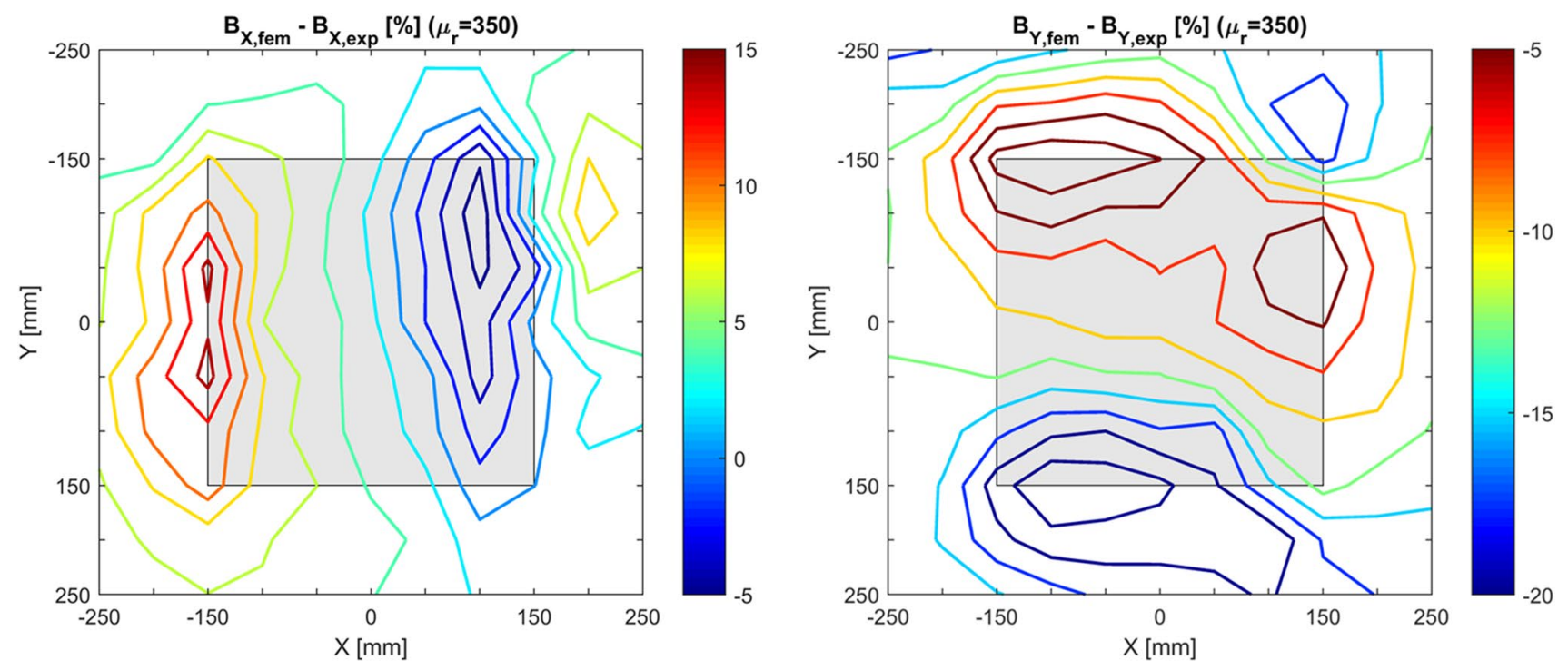

Fig. 21 Experiment 1-error distribution between FE results and measurements
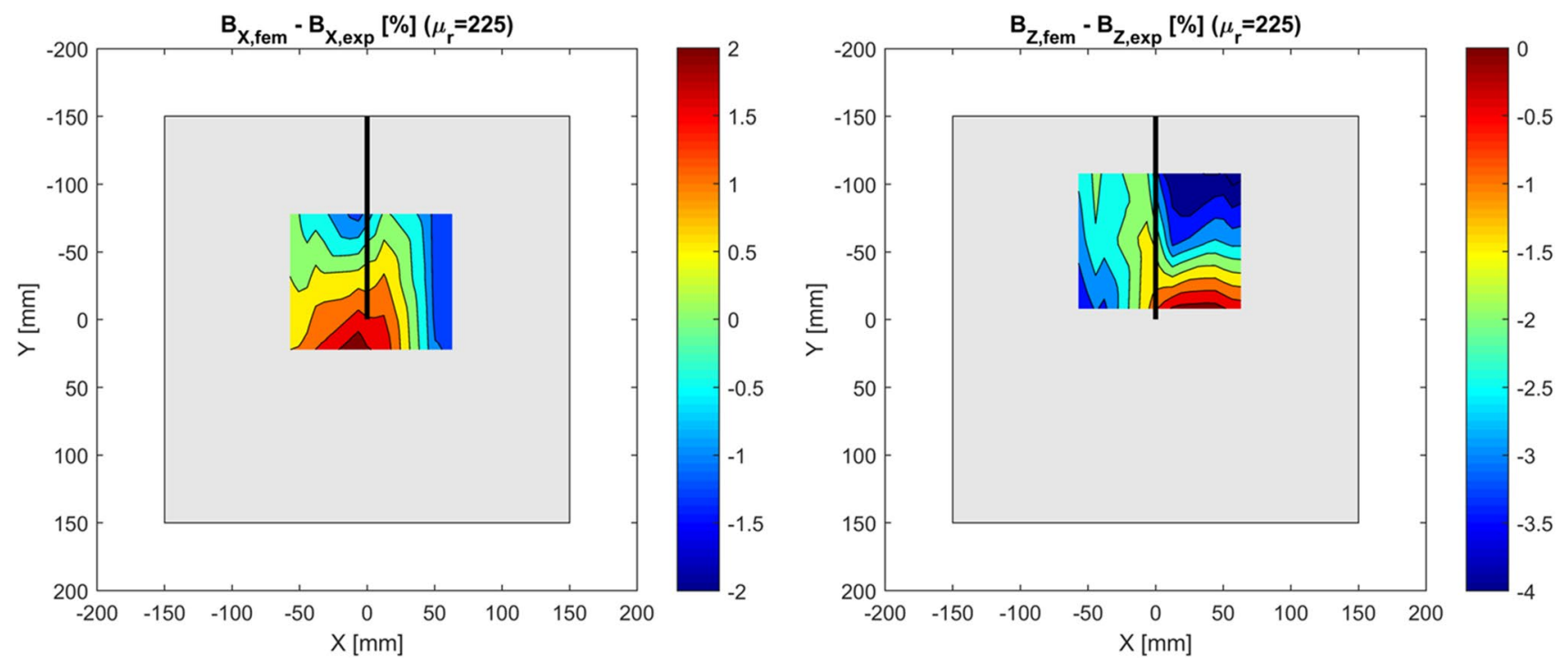

Fig. 22 Experiment 2-error distribution between FE results and measurements

the correct permanent magnetization for numerical simulation by FEM. At the same time, the measured permanent magnetic field distribution in Experiment 2 shows that for a plate with a slit, the permanent magnetization contributes significantly to the SMFL.

\section{Conclusions}

Being able to simulate novel crack monitoring methods based on the SMFL is important for the further development of these methods towards application on ship and offshore structures, ultimately leading to a reduction of operational expenses and increasing safety. The correct interpretation of the measured SMFL around a fatigue crack in a steel structure is needed to accurately size the crack. This involves thoroughly understanding all the sources that cause the SMFL, which can be subdivided into Earth induced magnetization and permanent magnetization. The aim of this paper was to determine the magnetic permeability of structural steels for simulation of the induced magnetic field distribution around cracks using FEM. 
Magnetic measurements were done above an FeE235 steel plate without defect (Experiment 1) and with a straight slit representing a fatigue crack (Experiment 2). The induced and permanent magnetic fields were separated using two different techniques. Comparing FE results with the measured induced magnetic fields suggests that the relative permeability of the plate from Experiment 1 is approximately 350 and that of Experiment 2 is approximately 225. Apparently, not every FeE235 steel plate has the same magnetic permeability. The error distributions between FE results and measurements suggest that the magnetic permeability of both plates are reasonably uniform. The measured permanent magnetic field in Experiment 1 confirms the hypothesis that the permanent magnetization of structural steel can be non-uniform. Its non-uniformity together with its relation to unknown stress and temperature histories make it challenging to model the permanent magnetization accurately.

Acknowledgements This work was funded by the CrackGuard Joint Industry Project, which is supported by Delft University of Technology and several industry partners (www.crack-guard.com). The authors would also like to acknowledge Aad Vijn and TNO for providing the hardware and support for the experimental part of this work.

Open Access This article is distributed under the terms of the Creative Commons Attribution 4.0 International License (http://creativeco mmons.org/licenses/by/4.0/), which permits unrestricted use, distribution, and reproduction in any medium, provided you give appropriate credit to the original author(s) and the source, provide a link to the Creative Commons license, and indicate if changes were made.

\section{References}

1. Van der Horst, M.P., Kaminski, M.L., Puik, E.: Methods for sensing and monitoring fatigue cracks and their applicability for marine structures. In: Proceedings on International Offshore Polar Engineering Conference, Anchorage, Alaska, pp. 455-462 (2013)

2. Dubov, A.: A study of metal properties using the method of magnetic memory. Met. Sci. Heat Treat. 39, 401-405 (1998)

3. Yao, K., Deng, B., Wang, Z.D.: Numerical studies to signal characteristics with the metal magnetic memory-effect in plastically deformed samples. NDT E Int. 47, 7-17 (2012). https:// doi.org/10.1016/j.ndteint.2011.12.004

4. Wang, Z.D., Yao, K., Deng, B., Ding, K.Q.: Theoretical studies of metal magnetic memory technique on magnetic flux leakage signals. NDT E Int. 43, 354-359 (2010). https://doi. org/10.1016/j.ndteint.2009.12.006

5. Wang, Z.D., Gu, Y., Wang, Y.S.: A review of three magnetic NDT technologies. J. Magn. Magn. Mater. 324, 382-388 (2012). https ://doi.org/10.1016/j.jmmm.2011.08.048
6. Jiles, D.C., Atherton, D.L.: Theory of ferromagnetic hysteresis (invited). J. Appl. Phys. 55, 2115-2120 (1984). https://doi. org/10.1063/1.333582

7. Davey, K.R.: Calculation of magnetic remanence. IEEE Trans. Magn. 45, 2907-2911 (2009). https://doi.org/10.1109/ TMAG.2009.2014562

8. Atherton, D.L., Jiles, D.C.: Effects of stress on magnetization. NDT Int. 19, 15-19 (1986). https://doi.org/10.1016/03089126(86)90135-5

9. Jiles, D.C., Devine, M.K.: The law of approach as a means of modelling the magnetomechanical effect. J. Magn. Magn. Mater. 140-144, 1881-1882 (1995). https://doi.org/10.1016/03048853(94)00928-7

10. Li, L., Jiles, D.C.: Modified law of approach for the magnetomechanical model: application of the Rayleigh law to stress. IEEE Trans. Magn. 39, 3037-3039 (2003). https://doi.org/10.1109/ TMAG.2003.815882

11. Bulte, D.P., Langman, R.A.: Origins of the magnetomechanical effect. J. Magn. Magn. Mater. 251, 229-243 (2002). https://doi. org/10.1016/s0304-8853(02)00588-7

12. Schwerer, F.C., Spangler, C.E., Kelly, J.F.: Temperature dependence of the magnetic coercivity of pearlite. Acta Metall. 26, 579589 (1978). https://doi.org/10.1016/0001-6160(78)90110-4

13. Kuz'min, M.D.: Shape of temperature dependence of spontaneous magnetization of ferromagnets: quantitative analysis. Phys. Rev. Lett. 94, 16-19 (2005). https://doi.org/10.1103/physrevlet t.94.107204

14. Van der Horst, M.P., Kaminski, M.L., Lepelaars, E.: Testing and numerical simulation of magnetic fields affected by presence of fatigue cracks. In: Proceeding on International Offshore Polar Engineering Conference, Busan, South Korea, pp. 445-450 (2014)

15. Hwang, J.H., Lord, W.: Finite element modeling of magnetic field/ defect interactions. ASTM J. Test. Eval. 3, 21-25 (1975)

16. Tanner, B.K., Szpunar, J.A., Willcock, S.N.M., Morgan, L.L., Mundell, P.A.: Magnetic and metallurgical properties of hightensile steels. J. Mater. Sci. 23, 4534-4540 (1988). https://doi. org/10.1007/BF00551956

17. Thompson, S.M., Tanner, B.K.: The magnetic properties of pearlitic steels as a function of carbon content. J. Magn. Magn. Mater. 123, 283-298 (1993). https://doi.org/10.1016/03048853(93)90454-A

18. Van der Horst, M.P., Kaminski, M.L.: Slit induced self magnetic flux leakage in a square steel plate. In: Proceedings on International Offshore Polar Engineering Conference, Sapporo, Japan, pp. 7-13 (2018)

19. Chung, H., Yang, C., Jung, W.: A magnetic field separation technique for a scaled model ship through an Earth's magnetic field simulator. J. Magn. 20, 62-68 (2015)

20. Somsen, O.J.G., Wagemakers, G.P.M.: Separating permanent and induced magnetic signature: a simple approach. Int. J. Electron. Comput. Energy Electron. Commun. Eng. 9, 1236-1239 (2015)

Publisher's Note Springer Nature remains neutral with regard to jurisdictional claims in published maps and institutional affiliations. 